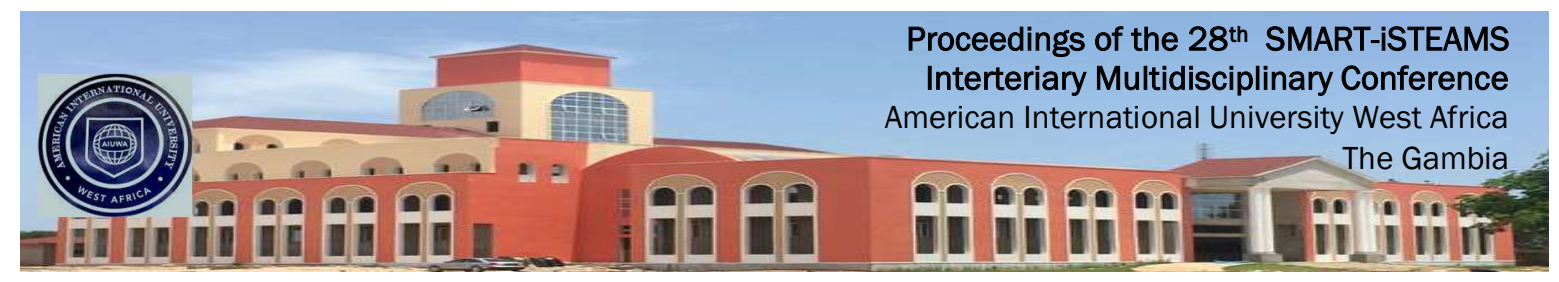

Full Research Paper

\title{
Strong Orthogonal Arrays and Orthogonal Array-Based Latin Hypercube Designs for Planning Experiments: A Method for Improving Study Design in Biomedical Research
}

\author{
10 suolale, Kazeem Adewale \\ 2Musa, Adesola Zaidat \\ 3Yahya, Waheed Babatunde \\ ${ }^{4}$ Otekunrin, Oluwaseun \\ Aramide \\ ${ }^{5}$ Babatunde Lateef Adeleke \\ 1\&2Nigerian Institute of \\ Medical Research \\ Yaba, Lagos \\ 3\&5University of Ilorin \\ Ilorin, Nigeria \\ 4University of Ibadan \\ Ibadan, Nigeria

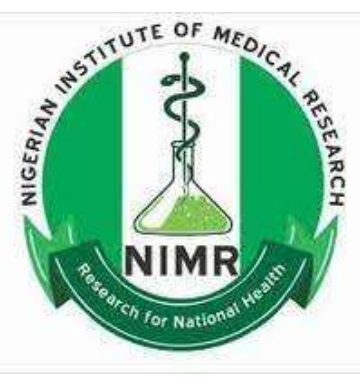

\section{E-mails} \\ ${ }^{1}$ whereisqosimadewale@gmail.com; \\ 2sola_dele@yahoo.com; \\ 3wbyahya@unilorin.edu.ng; \\ 4seramide2003@yahoo.co.uk \\ Phones \\ +2348066688932; \\ +2348033430791; \\ +2347030578626 ; \\ $+2348038357957$ \\ $+2348055401457$
}

\begin{abstract}
Orthogonal Array-based Latin Hypercube Designs (OALHDs) have not only become popular in practice among techniques used in the development of computer experiments but also helpful whenever interest is on designing some traditional experiments. Design construction for computer experiments has become a novel area especially in Nigeria and Africa at large since it is more about experimental planning rather than modelling aspect in which some progress has been made. The Bush Construction Type II method was used in this study to construct a strong Orthogonal Array (OA) of strength three, using Galois Fields (GF) of order s which gave rise to the constructed Orthogonal Array-Based Latin Hypercube Designs (OALHDs) for planning experiments. The OALHD was used in this research as a Latin hypercube design constructed based on orthogonal arrays in order to achieve better space-filling properties that would otherwise not be accomplished by a random Latin hypercube design (LHD). Orthogonal Array (N, k) LHD were constructed at parameter values of $\mathrm{OA}(\mathrm{N}, \mathrm{k})=(216,8)$ and $(343$, 9). This study aims at applying the OALHDs constructed to improve the study design or conducted in biomedical research. The health related quality of health data of HIV patients was used in the application. This is a novel approach in the medical setting. The method of construction employed the maximin criterion in the kNearest Neighbour with Euclidean distance for constructing strong the Orthogonal Array-Based Latin Hypercube Designs (OALHDs). The OA $(216,8)$ LHD and OA $(343,9)$ LHD constructed possessed better space-filling properties and they achieve uniformity in each dimension of the designed variables. This study concludes that the OALHDs can be used to improve the study design in biomedical research. A MATLAB 2016 computer package was used for program implementation and analysis.
\end{abstract}

Keywords: Computer experiments, Bush construction type II method, Galois fields, Latin hypercube designs, Orthogonal array

Proceedings Reference Format

Osuolale, K.A., Musa, A.Z. Yahya, W.B. ,Otekunrin, O.A. \& B.L. Adeleke (2021): Strong Orthogonal Arrays and Orthogonal Array-Based Latin Hypercube Designs for Planning Experiments: A Method for Improving Study Design in Biomedical Research. Proceedings of the 28th iSTEAMS Intertertiary Multidisciplinary Conference. American Int University West Africa, The Gambia. October, 2021. Pp 47-60. www.isteams.net/gambia2021.

DOI - https://doi.org/ 10.22624/AIMS/iSTEAMS-2021/V28N2P4 


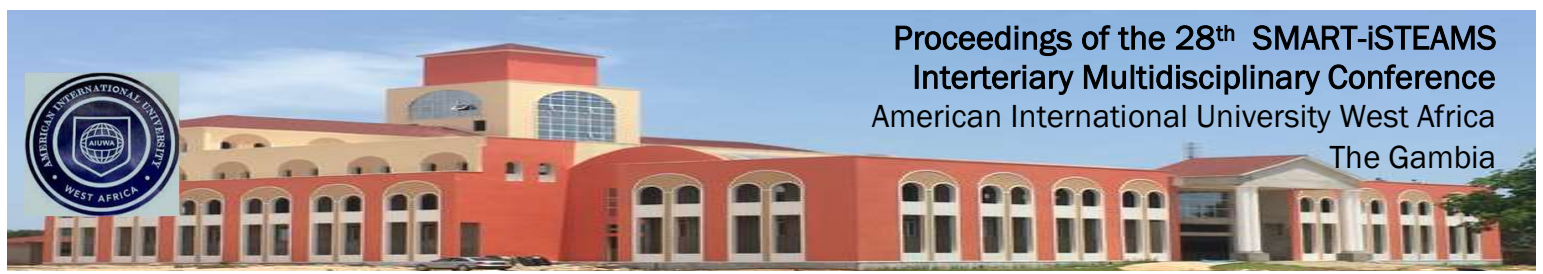

\section{INTRODUCTION}

Orthogonal arrays have been introduced due to their desirable statistical properties when used in fractional factorial experiments (Rao, 1947 and Bose and Bush, 1952). Although, other applications have been unveiled. Orthogonal arrays are not only useful in statistics but also useful in computer science and cryptography, medicine, agriculture and manufacturing. Owen (1992) recommended the use of orthogonal arrays as suitable designs for computer experiments; numerical integration and visualization since OAs ensure orthogonality, which guarantees that the input variables are uncorrelated. The applications of orthogonal arrays to statistical design of experiments discussed in Hedayat et al. (1999) are well known. Orthogonal arrays are greatly important in all areas of human investigations. Orthogonal arrays are used with Latin hypercube design to produce orthogonal array-based Latin hypercube designs to ensure better space-filling properties.

An orthogonal array of $\mathrm{N}$ runs, $k$ factors, $s$ levels, strength $t \geq 2$ and index $\lambda$ is an $n$-by- $k$ matrix with entries from a set of $s$ levels, usually taken as $0 . \ldots s-1$ such that for every $n$-by-k matrix of $s$ symbols, every subset of $t$ columns from among the $k$ columns, when considered alone must contain each of the possible $s^{t}$ ordered rows the same number of times. The variables $n, k, s, t$ and $\lambda$ are the parameters of the $O A$ and such an array is denoted by $O A(n, k, s, t)$. The parameter $\lambda\left(n / s^{t}\right)$ is referred to as the index parameter of the orthogonal array and is determined by the other four parameters. The most familiar examples of orthogonal arrays are regular fractional factorial designs discussed in Wu and Hamada (2000). The OA with $s_{1}=s_{2}=\cdots s_{n}=s$ is symmetric, otherwise, the $\mathrm{OA}$ is said to be asymmetric.

The rows of the array represent the experiments to be performed and the columns of the orthogonal array correspond to the different variables whose effects are being analyzed. The OALHDs constructed in this study is largely dependent on the existence of orthogonal arrays. The well known inequalities found by Rao (1947) for the construction of OAs have proferred a solution to this problem and one of the inequalities is adapted in this study.

Theorem 1: Rao's Inequalities

The Rao's inequalities are given by

$$
\begin{aligned}
& \text { (i) } \quad n \geq \sum_{i=0}^{u}\left(\begin{array}{l}
k \\
i
\end{array}\right)(s-1)^{i} \text {, if } t=2 u \text { and } \\
& \text { (ii) } n \geq \sum_{i=0}^{u}\left(\begin{array}{c}
k \\
i
\end{array}\right)(s-1)^{i}+\left(\begin{array}{c}
k-1 \\
u
\end{array}\right)(s-1)^{u+1} \text {, if } t=2 u+1 \text { for } u \geq 0
\end{aligned}
$$

This theorem provides a scheme for determining either a lower bound on the number of rows, $n$, in any OA $(n, k, s, t)$ design for given values, $k, s$ and $t$, or an upper bound on the number of columns, $k$, for given values, $n, s$ and $t$ (Osuolale, 2017). The proof of this theorem is given in Hedayat et al. (1999). The use of these inequalities depends on whether $t$ is even or odd. Orthogonal Array-Based Latin Hypercube Design (OALHD) is a Latin hypercube design constructed based on orthogonal arrays in order to maintain the univariate stratification and achieve better space-filling properties. Space-filling designs are designs that spread design points evenly throughout the experimental region. Space-filling designs prevent replicate points by spreading the design points out to the maximum distance possible between any two points and distribute the points uniformly. It is known that not all Latin hypercube designs are good 


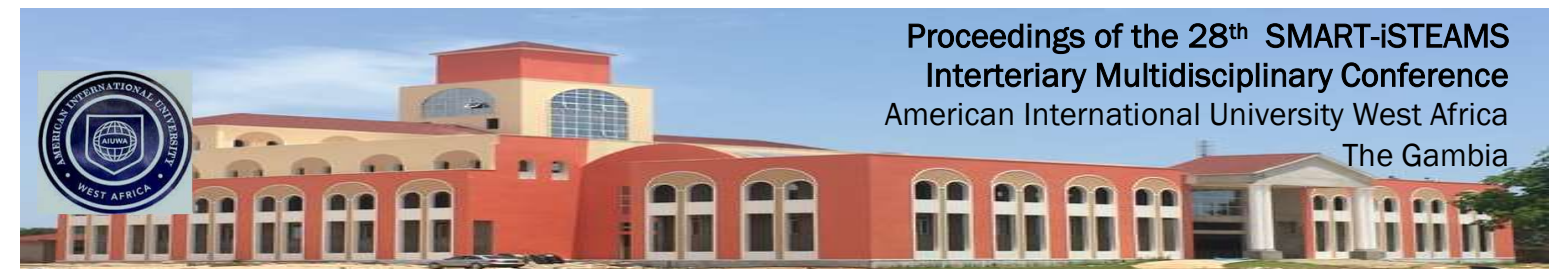

and hence OAs and LHDs have been hybridized to have OALHDs using the approach discussed in this study. Several experimental space-filling criteria have been proposed in the literature to construct space-filling designs that give good coverage of the design space and offer low correlation among the design points. Orthogonal designs offer uncorrelated input variables that help to independently assess the effect of individual input variable on the response. These two properties are important characteristics of good experimental designs for planning experiments. Maximin and Minimax Criteria are such criteria that guarantee space-filling properties.

Maximin and Minimax Criteria were originally proposed by Johnson et al. (1990) for use in the development of computer experiments. Shewry and Wynn (1988) and Currin et al. (1991) use the maximum entropy principle to develop designs for developing computer experiments. An optimal Latin hypercube design for computer experiments which either minimizes Integrated Mean Squared Error (IMSE) or maximizes entropy has also been discussed by Park (1994).

Tang (1993) proposed OALHDs that are more suitable for planning or designing computer experiments than general Latin hypercube designs. He started his construction with an OA $\left(s^{2}\right.$, $k, s)$, and then replaced the $s$ positions with symbol $t$ by a random permutation of $(t-1) s+1$, . $\ldots, t s$, for all $t=1, \ldots, s$. After the replacement procedure was done for all the $k$ columns, the resulting matrix was denoted by $D=\left(d_{i j}\right), i=1, \ldots, s^{2}, j=1, \ldots, k$ which forms an $s^{2}$ $x k$ OALHD with $s^{2}$ levels. Leary et al. (2003) considered searching for optimal OA-based Latin hypercubes using an alternative distance metric that minimizes

$$
\sum_{i=1}^{n} \sum_{j=i+1}^{n} \frac{1}{d_{i j}^{2}}
$$

where

' $n$ ' is the number of sampled points and

$d_{i j}$ is the distance between points

$i$ and $j$ and is defined as:

$$
d_{i j}=\frac{l_{i j}{ }^{(n-1)} / 2^{+} u_{i j}}{n}, i=1, \ldots n, j=1, \ldots k
$$

This criterion is used to search for a restricted subspace of the set of all OALHDs. Leary et al. (2003) adapted strategies found in Morris and Mitchell (1995) and Ye et al. (2000) by performing optimization using the simulated annealing and the columnwise-pairwise algorithms.

Qian et al. (2006) also proposed a method for constructing OALHDs as nested space-filling designs for multiple experiments with different levels of accuracy. They considered two experiments called low-accuracy experiment (LE) and high-accuracy experiment (HE). Their construction used a 2-step procedure. 


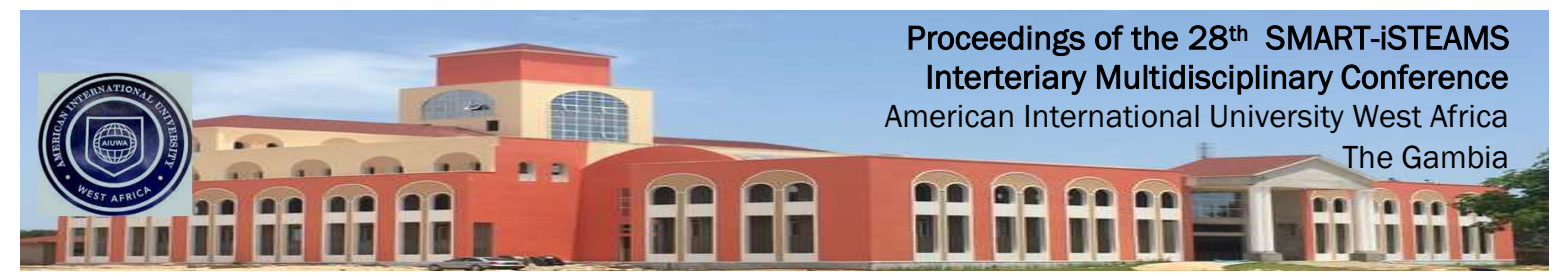

The first step constructs an OALHD for $D_{1}$ with size $n_{1}$ and the second step chooses a subset of $D_{1}$ with size $n_{2}$ as $D_{h}$ based on the maximin distance criterion using

$$
D_{h}=\arg \max _{D}\left[\min _{x_{i}, x_{j} \in D} \mathrm{~d}\left(\mathbf{x}_{i}, \mathbf{x}_{j}\right)\right]
$$

where

$D$ is any subset of $D$, with size $n_{2}$.

OALHD appeared to be a good choice for $D_{l}$ but $D_{h}$ is far from being space-filling in this procedure. Qian et al. (2009) used nested orthogonal arrays and nested difference matrices to achieve space-filling and maximum stratification for both $D_{h}$ and $D_{1}$ to mitigate the drawbacks in the non space-filling property of $D_{h}$ earlier constructed by Qian et al. (2006). For details of LHDs that are good based on some optimal design criteria, Iman and Conover (1982), Owen (1994), Morris and Mitchell (1995), Ye (1998), Ye et al. (2000), Jin et al. (2005), Joseph et al. (2008) and Hernandez et al. (2012) can be consulted.

Osuolale et al. (2014) proposed a technique for the construction of space-filling designs for three input variables computer experiments. The technique limits the number of input variables to three with different number of runs. Yahya and Osuolale (2016) also proposed a method of constructing OALHDs for planning computer experiments. Yahya and Osuolale (2016) used an improved technique to construct OALHDs. The maximin distance criterion employed in this current study to obtain the optimal designs made use of KNN search with Euclidean distance that finds the nearest neighbour in $L$ for each point in $L$.

\section{MATERIAL AND METHODS}

A MATLAB 2016 software was used to construct Orthogonal Array-Based Latin Hypercube Designs (OALHDs). A mathematical theorem was adopted in the construction of OALHDs and the desired OALHDs were optimized using a maximin distance criterion. The OALHD algorithm employed the maximin distance criterion in a unique way using the $k$-Nearest Neighbour (KNN) search with Euclidean distance to maximize the minimum distance between any two design points from the possible design points of $(n !)^{k}$ in order to obtain the optimal design with spacefilling properties. These techniques searched only for OALHDs and consequently, optimal designs that are guaranteed to have some space-filling properties were constructed. The OALHD main function is given as:

$$
[D / L]=f(\text { level, strength })
$$

An uppercase character $D$ represents Orthogonal Array (OA), $L$ is the desired OALHD, factors $\left\{x_{1}, x_{2}, \ldots, x_{n}\right\}$ are the input variables and the level $\{0,1,2, \ldots, s-1\}$ represents a set of entries used in the orthogonal array to construct the design. This method is based on the use of Galois fields. A field is composed of a set, $F$, and two binary operations that map $F X F$ into $F$. 


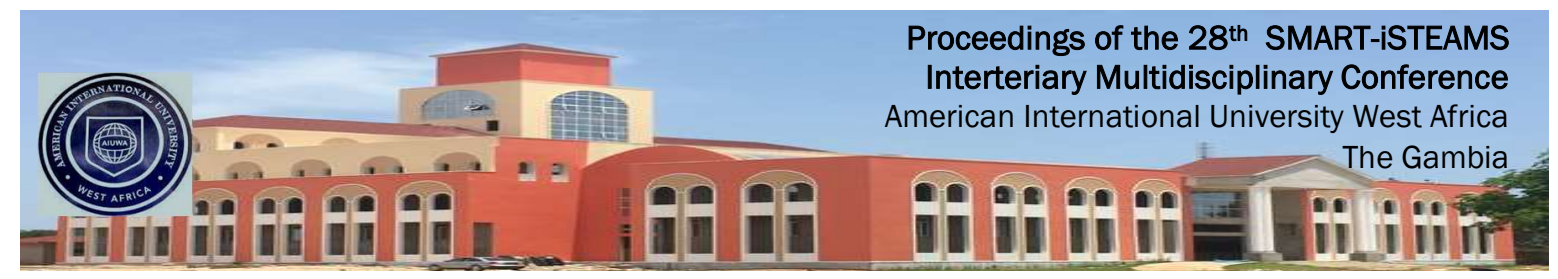

A simple example is the set of positive integers along with the operations of ordinary addition and multiplication. A Galois field is one for which the set, $\mathrm{F}$, is finite.

Theorem 2: Orthogonal Arrays using Bush Construction Type II method (Hedayat et al., 1999) If $s=2^{m}, m \geq 1$ and $\mathrm{t}=3$ then there exists an $O A\left(s^{t}, s+2, s, t\right)$ of index unity. This technique works for OALHDs of large number of runs and different number of input variables and $s$ levels with either even or odd numbers.

Having constructed the $\mathrm{OA}$, ranking is done such that:

i. Each column of the $\mathrm{OA}$ is sorted in ascending order

ii. The sorting order is used to create a set taken from $S=\{1,2, \ldots, n\}$

iii. It returns a column vector of $S$ as $d_{i}$.

Therefore:

$R=\left[d_{1} d_{2} \ldots d_{m}\right]$

With this, an initial OALHD is created as:

$L=\frac{d-0.1}{n}$

Where

$n$ is given as $n=s^{3}$.

To add space filling properties, the design, $L$, is optimised using a choice of maximin distance criterion as follows:

i. A new design is created as follows:

$$
L_{\text {new }}=\frac{d-u_{i j}}{n}
$$

where

$u_{i j}$ is taken from a uniform distribution [0 1] and

$\mathrm{d}$ from the OA earlier constructed.

ii. The new design is scored based on k-Nearest Neighbours using Euclidean distance which finds the nearest neighbour in $L$ for each point in $L$. The algorithm is based on:

$$
d_{s t}^{2}=\left(L_{s}-L_{t}\right)\left(L_{s}-L_{t}\right)^{\prime}
$$




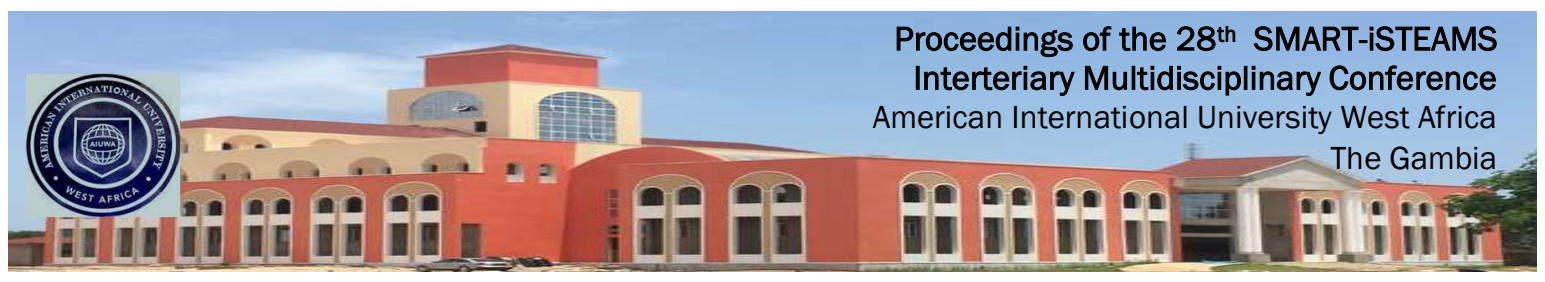

The $d_{s t}$ is the distance between $L_{s}$ and $L_{t}$. Since two nearest neighbours are required, $d_{s t}$ is returned as a two-column matrix of which the second column is extracted as the maximum between the two columns since the first column contains zero all through. Then the extracted column is scored by minimizing the distance between the entries in the column using:

$$
\text { score }=\min \left(d_{s t_{2}}\right)
$$

where $d_{s t_{2}}$ is the extracted second column.

iii. An iteration is performed to determine the best design based on maximin criterion as follows:

$$
\begin{aligned}
& \text { Score }_{\text {old }}=\operatorname{score}(L) \\
& \text { Score }_{\text {new }}=\operatorname{score}\left(L_{\text {new }}\right) \\
& L=\left\{\begin{array}{c}
L_{\text {new }} \text { if } \text { Score }_{\text {new }}>\text { Score }_{\text {old }} \\
\text { Otherwise }
\end{array}\right. \\
& \text { Score }_{\text {old }}=\left\{\begin{array}{cc}
\text { Score }_{\text {new }} \text { Score }_{\text {new }}>\text { Score }_{\text {old }} \\
\text { Score }_{\text {old }} & \text { Otherwise }
\end{array}\right.
\end{aligned}
$$

\section{RESULTS}

The results of the orthogonal array-based Latin hypercube designs, OA $(216,8)$ LHD and OA $(343,9)$ LHD constructed from OA $(216,8,6,3)$ and OA $(343,9,7,3)$ are provided in Table 1 and Table 2 with $[D, L]=$ oa_test2 $(6,3)$ and oa_test2 $(9,3)$, respectively. The plots for the projections of design points among various input variables are given in Figures 1 and 2, respectively. 


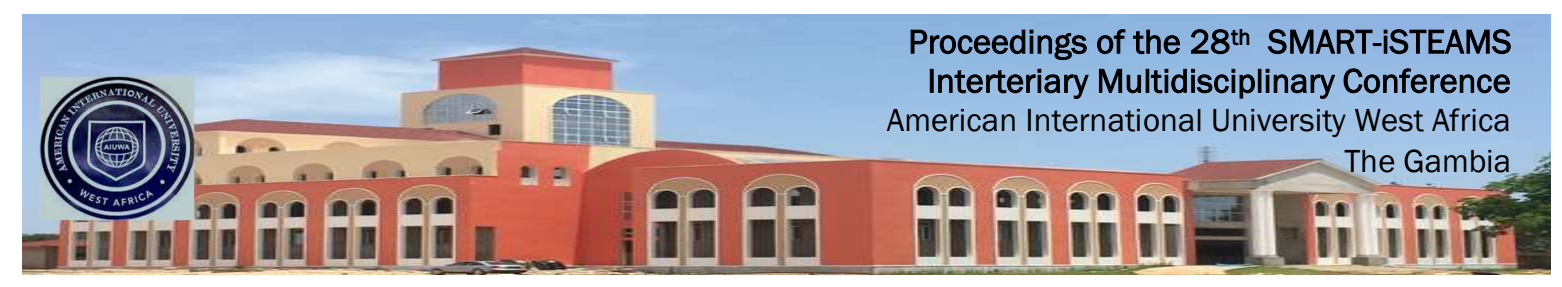

Table 1: OA $(216,8)$ LHD

$[D, L]=0 a \_t e s t 2(6,3)$

\begin{tabular}{|c|c|c|c|c|c|c|c|c|}
\hline \multicolumn{9}{|c|}{ Design Points (OALHD) } \\
\hline No. & $X_{1}$ & $X_{2}$ & $X_{3}$ & $\mathrm{X}_{4}$ & $x_{5}$ & $X_{6}$ & $\mathrm{X}_{7}$ & $X_{8}$ \\
\hline 1 & 0.004 & 0.004 & 0.004 & 0.004 & 0.004 & 0.004 & 0.004 & 0.004 \\
\hline 2 & 0.009 & 0.134 & 0.282 & 0.351 & 0.213 & 0.143 & 0.171 & 0.009 \\
\hline 3 & 0.013 & 0.263 & 0.560 & 0.671 & 0.421 & 0.282 & 0.338 & 0.013 \\
\hline 4 & 0.018 & 0.393 & 0.782 & 0.949 & 0.351 & 0.421 & 0.504 & 0.018 \\
\hline 5 & 0.023 & 0.523 & 0.213 & 0.490 & 0.782 & 0.560 & 0.671 & 0.023 \\
\hline 6 & 0.027 & 0.652 & 0.490 & 0.143 & 0.949 & 0.671 & 0.838 & 0.027 \\
\hline 7 & 0.032 & 0.138 & 0.143 & 0.213 & 0.282 & 0.351 & 0.009 & 0.171 \\
\hline 8 & 0.037 & 0.009 & 0.421 & 0.421 & 0.490 & 0.490 & 0.175 & 0.175 \\
\hline 9 & 0.041 & 0.398 & 0.671 & 0.615 & 0.143 & 0.074 & 0.342 & 0.180 \\
\hline 10 & 0.046 & 0.268 & 0.893 & 0.782 & 0.074 & 0.213 & 0.509 & 0.185 \\
\hline 11 & 0.050 & 0.657 & 0.074 & 0.282 & 0.560 & 0.838 & 0.675 & 0.189 \\
\hline 12 & 0.055 & 0.527 & 0.351 & 0.074 & 0.726 & 0.949 & 0.842 & 0.194 \\
\hline 13 & 0.060 & 0.273 & 0.287 & 0.425 & 0.564 & 0.675 & 0.013 & 0.338 \\
\hline 14 & 0.064 & 0.402 & 0.009 & 0.217 & 0.731 & 0.564 & 0.180 & 0.342 \\
\hline 15 & 0.069 & 0.013 & 0.787 & 0.787 & 0.893 & 0.893 & 0.347 & 0.347 \\
\hline 16 & 0.074 & 0.143 & 0.564 & 0.620 & 0.838 & 0.782 & 0.513 & 0.351 \\
\hline 17 & 0.078 & 0.782 & 0.495 & 0.078 & 0.287 & 0.148 & 0.680 & 0.356 \\
\hline 18 & 0.083 & 0.893 & 0.217 & 0.287 & 0.495 & 0.009 & 0.847 & 0.361 \\
\hline 19 & 0.088 & 0.407 & 0.425 & 0.356 & 0.787 & 0.953 & 0.018 & 0.504 \\
\hline 20 & 0.092 & 0.277 & 0.148 & 0.009 & 0.953 & 0.842 & 0.185 & 0.509 \\
\hline 21 & 0.097 & 0.148 & 0.898 & 0.953 & 0.671 & 0.726 & 0.351 & 0.513 \\
\hline 22 & 0.101 & 0.018 & 0.675 & 0.675 & 0.615 & 0.615 & 0.518 & 0.518 \\
\hline 23 & 0.106 & 0.898 & 0.356 & 0.148 & 0.009 & 0.495 & 0.685 & 0.523 \\
\hline 24 & 0.111 & 0.787 & 0.078 & 0.495 & 0.217 & 0.356 & 0.851 & 0.527 \\
\hline 25 & 0.115 & 0.532 & 0.569 & 0.791 & 0.222 & 0.500 & 0.023 & 0.671 \\
\hline 26 & 0.120 & 0.662 & 0.791 & 0.625 & 0.013 & 0.361 & 0.189 & 0.675 \\
\hline 27 & 0.125 & 0.791 & 0.013 & 0.430 & 0.356 & 0.217 & 0.356 & 0.680 \\
\hline 28 & 0.129 & 0.902 & 0.291 & 0.222 & 0.425 & 0.078 & 0.523 & 0.685 \\
\hline 29 & 0.134 & 0.023 & 0.726 & 0.726 & 0.958 & 0.958 & 0.689 & 0.689 \\
\hline 30 & 0.138 & 0.152 & 0.949 & 0.893 & 0.791 & 0.847 & 0.856 & 0.694 \\
\hline 31 & 0.143 & 0.666 & 0.680 & 0.958 & 0.500 & 0.152 & 0.027 & 0.838 \\
\hline 32 & 0.148 & 0.537 & 0.902 & 0.680 & 0.291 & 0.013 & 0.194 & 0.842 \\
\hline 33 & 0.152 & 0.907 & 0.152 & 0.361 & 0.078 & 0.425 & 0.361 & 0.847 \\
\hline 34 & 0.157 & 0.796 & 0.430 & 0.013 & 0.148 & 0.287 & 0.527 & 0.851 \\
\hline 35 & 0.162 & 0.157 & 0.615 & 0.560 & 0.736 & 0.680 & 0.694 & 0.856 \\
\hline 36 & 0.166 & 0.027 & 0.838 & 0.838 & 0.569 & 0.569 & 0.861 & 0.861 \\
\hline 37 & 0.171 & 0.162 & 0.083 & 0.083 & 0.083 & 0.083 & 0.032 & 0.032 \\
\hline 38 & 0.175 & 0.032 & 0.361 & 0.291 & 0.152 & 0.222 & 0.199 & 0.037 \\
\hline . & & & ". & . & & . & . & \\
\hline
\end{tabular}




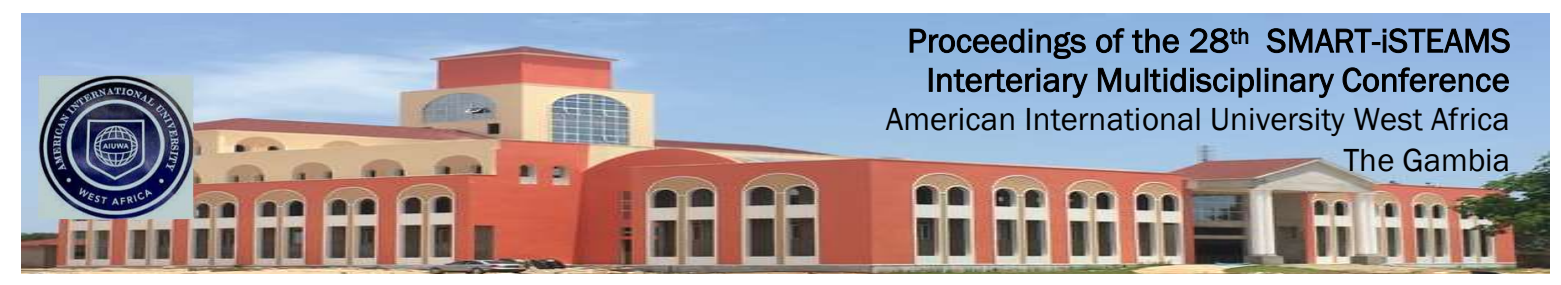

\begin{tabular}{|r|r|r|r|r|r|r|r|r|}
\hline. &. &. &. &. &. &. &. &. \\
\hline. &. &. &. &. &. &. &. &. \\
\hline. &. &. &. &. &. &. &. &. \\
\hline. &. &. &. &. &. &. &. &. \\
\hline. &. &. &. &. &. &. &. &. \\
\hline. &. &. &. &. &. &. &. &. \\
\hline. &. &. &. &. &. &. &. &. \\
\hline. &. &. &. &. &. &. &. &. \\
\hline 200 & 0.925 & 1.000 & 0.550 & 0.412 & 0.717 & 0.606 & 0.324 & 0.648 \\
\hline 201 & 0.930 & 0.638 & 0.773 & 0.717 & 1.000 & 0.944 & 0.490 & 0.652 \\
\hline 202 & 0.935 & 0.768 & 0.995 & 0.995 & 0.833 & 0.833 & 0.657 & 0.657 \\
\hline 203 & 0.939 & 0.379 & 0.069 & 0.555 & 0.412 & 0.203 & 0.824 & 0.662 \\
\hline 204 & 0.944 & 0.509 & 0.347 & 0.208 & 0.481 & 0.064 & 0.990 & 0.666 \\
\hline 205 & 0.949 & 0.254 & 0.888 & 0.666 & 0.486 & 0.208 & 0.162 & 0.810 \\
\hline 206 & 0.953 & 0.125 & 0.666 & 0.833 & 0.416 & 0.069 & 0.328 & 0.814 \\
\hline 207 & 0.958 & 0.513 & 0.416 & 0.277 & 0.069 & 0.486 & 0.495 & 0.819 \\
\hline 208 & 0.963 & 0.384 & 0.138 & 0.486 & 0.277 & 0.347 & 0.662 & 0.824 \\
\hline 209 & 0.967 & 0.773 & 0.944 & 0.944 & 0.722 & 0.722 & 0.828 & 0.828 \\
\hline 210 & 0.972 & 0.643 & 0.722 & 0.777 & 0.666 & 0.611 & 0.995 & 0.833 \\
\hline 211 & 0.976 & 0.129 & 1.000 & 0.722 & 0.208 & 0.555 & 0.166 & 0.976 \\
\hline 212 & 0.981 & 0.259 & 0.777 & 1.000 & 0.138 & 0.416 & 0.333 & 0.981 \\
\hline 213 & 0.986 & 0.388 & 0.555 & 0.069 & 0.347 & 0.277 & 0.500 & 0.986 \\
\hline 214 & 0.990 & 0.518 & 0.277 & 0.416 & 0.555 & 0.138 & 0.666 & 0.990 \\
\hline 215 & 0.995 & 0.648 & 0.833 & 0.888 & 0.944 & 1.000 & 0.833 & 0.995 \\
\hline 216 & 1.000 & 0.777 & 0.611 & 0.611 & 0.888 & 0.888 & 1.000 & 1.000 \\
\hline
\end{tabular}

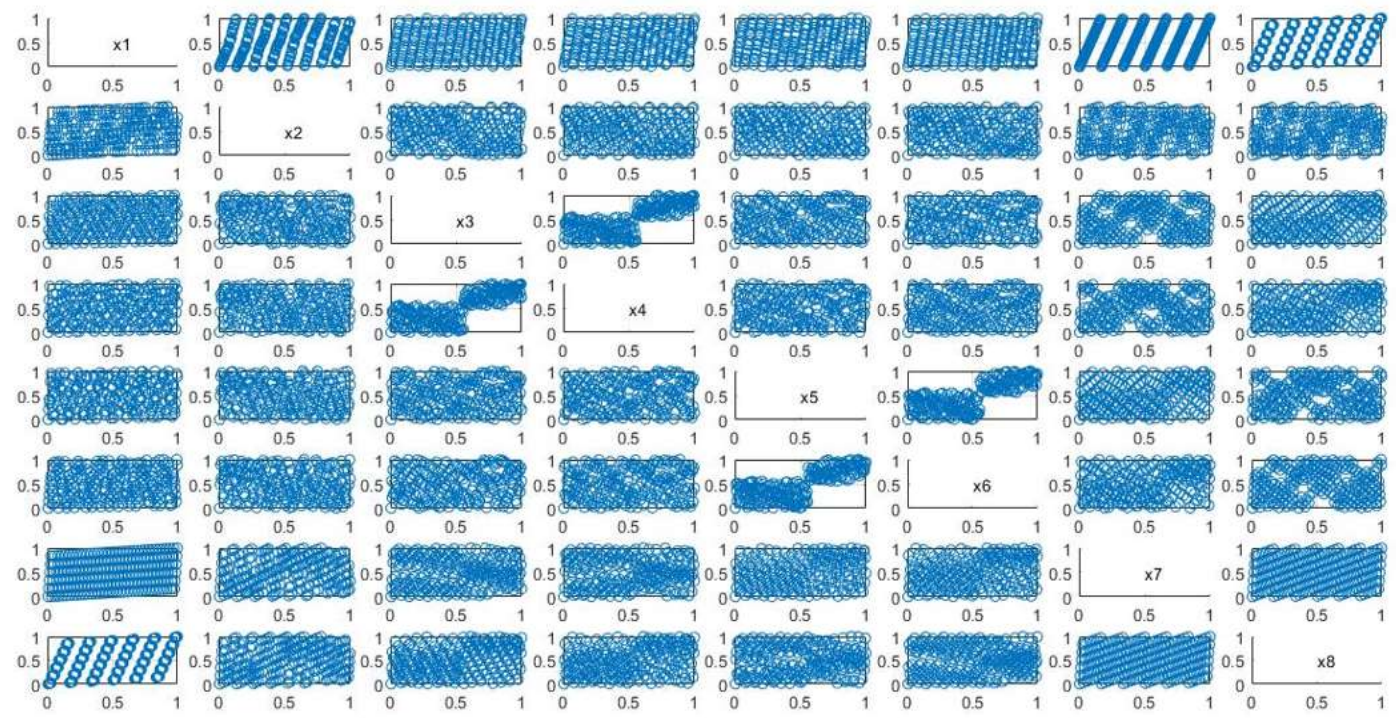

Figure 1: Projection properties of OA $(216,8)$ LHD 


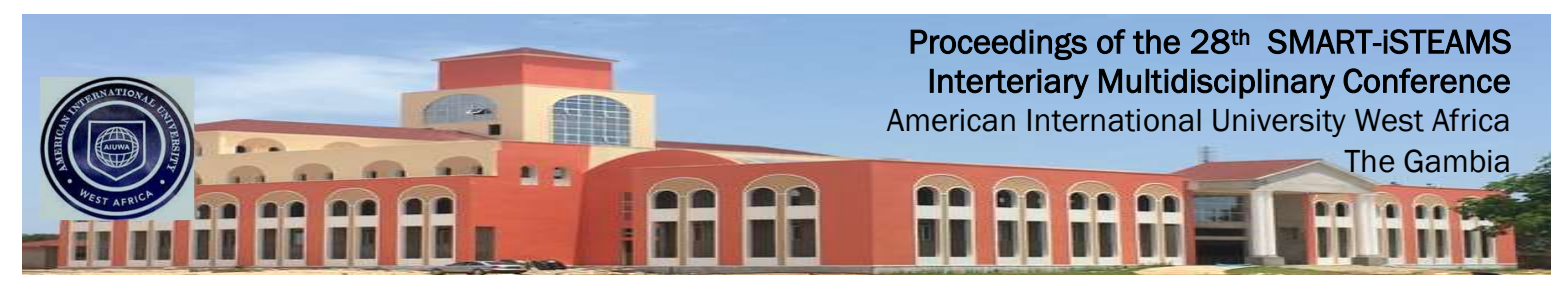

Table 2: OA $(343,9)$ LHD

$[D, L]=0 a \_t e s t 2(7,3)$

\begin{tabular}{|c|c|c|c|c|c|c|c|c|c|}
\hline \multicolumn{10}{|c|}{ Design Points (OALHD) } \\
\hline No. & $X_{1}$ & $x_{2}$ & $x_{3}$ & $\mathrm{X}_{4}$ & $x_{5}$ & $\mathrm{X}_{6}$ & $x_{7}$ & $X_{8}$ & $X_{9}$ \\
\hline 1 & 0.003 & 0.003 & 0.003 & 0.003 & 0.003 & 0.003 & 0.003 & 0.003 & 0.003 \\
\hline 2 & 0.006 & 0.128 & 0.145 & 0.178 & 0.513 & 0.545 & 0.624 & 0.145 & 0.006 \\
\hline 3 & 0.008 & 0.253 & 0.288 & 0.358 & 0.172 & 0.236 & 0.399 & 0.288 & 0.008 \\
\hline 4 & 0.011 & 0.379 & 0.431 & 0.533 & 0.670 & 0.699 & 0.790 & 0.431 & 0.011 \\
\hline 5 & 0.014 & 0.504 & 0.574 & 0.688 & 0.329 & 0.452 & 0.819 & 0.574 & 0.014 \\
\hline 6 & 0.017 & 0.629 & 0.688 & 0.600 & 0.819 & 0.971 & 0.428 & 0.717 & 0.017 \\
\hline 7 & 0.020 & 0.755 & 0.799 & 0.950 & 0.484 & 0.297 & 0.726 & 0.860 & 0.020 \\
\hline 8 & 0.023 & 0.131 & 0.076 & 0.105 & 0.137 & 0.172 & 0.189 & 0.006 & 0.145 \\
\hline 9 & 0.026 & 0.006 & 0.218 & 0.218 & 0.635 & 0.635 & 0.559 & 0.148 & 0.148 \\
\hline 10 & 0.029 & 0.382 & 0.361 & 0.317 & 0.038 & 0.070 & 0.338 & 0.291 & 0.151 \\
\hline 11 & 0.032 & 0.256 & 0.504 & 0.431 & 0.545 & 0.606 & 0.973 & 0.434 & 0.154 \\
\hline 12 & 0.035 & 0.632 & 0.632 & 0.766 & 0.452 & 0.358 & 0.883 & 0.577 & 0.157 \\
\hline 13 & 0.038 & 0.507 & 0.743 & 0.632 & 0.941 & 0.816 & 0.248 & 0.720 & 0.160 \\
\hline 14 & 0.041 & 0.880 & 0.851 & 0.921 & 0.358 & 0.390 & 0.527 & 0.863 & 0.163 \\
\hline 15 & 0.043 & 0.259 & 0.148 & 0.221 & 0.268 & 0.326 & 0.367 & 0.008 & 0.288 \\
\hline 16 & 0.046 & 0.385 & 0.006 & 0.108 & 0.758 & 0.848 & 0.758 & 0.151 & 0.291 \\
\hline 17 & 0.049 & 0.008 & 0.434 & 0.434 & 0.422 & 0.420 & 0.032 & 0.294 & 0.294 \\
\hline 18 & 0.052 & 0.134 & 0.291 & 0.320 & 0.912 & 0.880 & 0.656 & 0.437 & 0.297 \\
\hline 19 & 0.055 & 0.758 & 0.691 & 0.635 & 0.073 & 0.137 & 0.691 & 0.580 & 0.300 \\
\hline 20 & 0.058 & 0.883 & 0.577 & 0.769 & 0.577 & 0.664 & 0.064 & 0.723 & 0.303 \\
\hline 21 & 0.061 & 0.510 & 0.901 & 0.799 & 0.236 & 0.102 & 0.851 & 0.866 & 0.306 \\
\hline 22 & 0.064 & 0.387 & 0.221 & 0.180 & 0.390 & 0.484 & 0.309 & 0.011 & 0.431 \\
\hline 23 & 0.067 & 0.262 & 0.078 & 0.006 & 0.880 & 0.941 & 0.944 & 0.154 & 0.434 \\
\hline 24 & 0.070 & 0.137 & 0.507 & 0.536 & 0.300 & 0.268 & 0.218 & 0.297 & 0.437 \\
\hline 25 & 0.073 & 0.011 & 0.364 & 0.361 & 0.790 & 0.787 & 0.592 & 0.440 & 0.440 \\
\hline 26 & 0.076 & 0.886 & 0.746 & 0.603 & 0.204 & 0.035 & 0.492 & 0.583 & 0.443 \\
\hline 27 & 0.078 & 0.761 & 0.635 & 0.691 & 0.699 & 0.513 & 0.128 & 0.726 & 0.446 \\
\hline 28 & 0.081 & 0.635 & 0.953 & 0.871 & 0.105 & 0.204 & 0.915 & 0.869 & 0.449 \\
\hline 29 & 0.084 & 0.513 & 0.294 & 0.437 & 0.516 & 0.638 & 0.761 & 0.014 & 0.574 \\
\hline 30 & 0.087 & 0.638 & 0.437 & 0.323 & 0.006 & 0.175 & 0.370 & 0.157 & 0.577 \\
\hline 31 & 0.090 & 0.764 & 0.008 & 0.224 & 0.673 & 0.609 & 0.659 & 0.300 & 0.580 \\
\hline 32 & 0.093 & 0.889 & 0.151 & 0.110 & 0.175 & 0.073 & 0.035 & 0.443 & 0.583 \\
\hline 33 & 0.096 & 0.014 & 0.801 & 0.801 & 0.822 & 0.819 & 0.067 & 0.586 & 0.586 \\
\hline 34 & 0.099 & 0.140 & 0.903 & 0.924 & 0.332 & 0.361 & 0.694 & 0.729 & 0.589 \\
\hline 35 & 0.102 & 0.265 & 0.580 & 0.638 & 0.973 & 0.912 & 0.460 & 0.871 & 0.592 \\
\hline 36 & 0.105 & 0.641 & 0.367 & 0.539 & 0.638 & 0.548 & 0.947 & 0.017 & 0.717 \\
\hline 37 & 0.108 & 0.516 & 0.510 & 0.364 & 0.140 & 0.006 & 0.312 & 0.160 & 0.720 \\
\hline 38 & 0.110 & 0.892 & 0.081 & 0.183 & 0.548 & 0.702 & 0.594 & 0.303 & 0.723 \\
\hline . & . & . & . & . & . & . &. & . & \\
\hline & & & & & & & & & \\
\hline
\end{tabular}




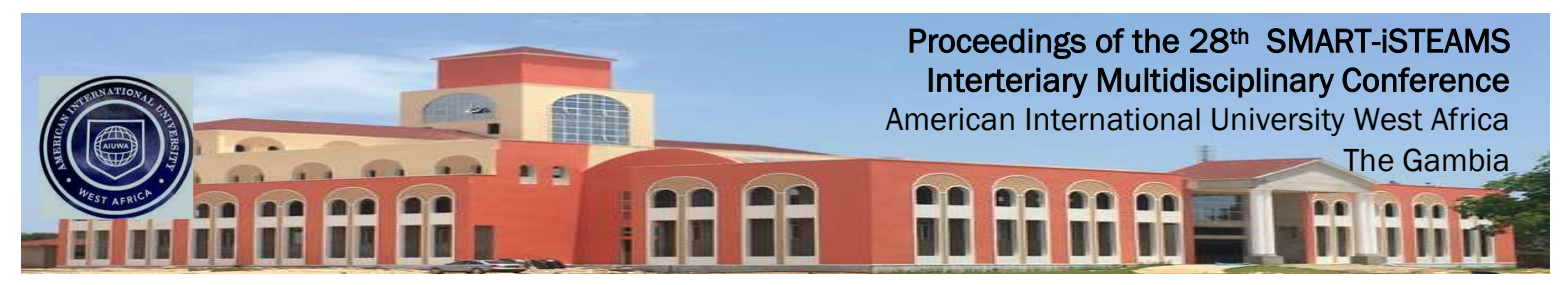

\begin{tabular}{|r|r|r|r|r|r|r|r|r|r|}
\hline. &. &. &. &. &. &. &. &. & \\
\hline. &. &. &. &. &. &. &. &. & \\
\hline. &. &. &. &. &. &. &. &. & \\
\hline. &. &. &. &. &. &. &. &. & \\
\hline. &. &. &. &. &. &. &. &. & \\
\hline. &. &. &. &. &. &. &. &. & \\
\hline. &. &. &. &. &. &. &. &. & \\
\hline 327 & 0.953 & 0.871 & 0.976 & 0.976 & 0.909 & 0.909 & 0.157 & 0.708 & 0.708 \\
\hline 328 & 0.956 & 0.997 & 0.874 & 0.898 & 0.420 & 0.449 & 0.524 & 0.851 & 0.711 \\
\hline 329 & 0.959 & 0.624 & 0.772 & 0.717 & 0.816 & 0.877 & 0.306 & 0.994 & 0.714 \\
\hline 330 & 0.962 & 0.498 & 0.469 & 0.352 & 0.603 & 0.755 & 0.787 & 0.140 & 0.839 \\
\hline 331 & 0.965 & 0.373 & 0.326 & 0.466 & 0.102 & 0.233 & 0.396 & 0.283 & 0.842 \\
\hline 332 & 0.968 & 0.248 & 0.183 & 0.140 & 0.755 & 0.542 & 0.688 & 0.425 & 0.845 \\
\hline 333 & 0.971 & 0.122 & 0.041 & 0.253 & 0.265 & 0.067 & 0.061 & 0.568 & 0.848 \\
\hline 334 & 0.973 & 1.000 & 0.927 & 0.944 & 0.787 & 0.813 & 0.096 & 0.711 & 0.851 \\
\hline 335 & 0.976 & 0.874 & 0.825 & 0.822 & 0.297 & 0.294 & 0.723 & 0.854 & 0.854 \\
\hline 336 & 0.979 & 0.749 & 0.717 & 0.796 & 0.938 & 0.968 & 0.490 & 0.997 & 0.857 \\
\hline 337 & 0.982 & 0.125 & 0.399 & 0.469 & 0.971 & 0.784 & 0.589 & 0.143 & 0.982 \\
\hline 338 & 0.985 & 0.250 & 0.542 & 0.355 & 0.481 & 0.323 & 0.215 & 0.285 & 0.985 \\
\hline 339 & 0.988 & 0.376 & 0.113 & 0.256 & 0.877 & 1.000 & 1.000 & 0.428 & 0.988 \\
\hline 340 & 0.991 & 0.501 & 0.256 & 0.143 & 0.387 & 0.481 & 0.364 & 0.571 & 0.991 \\
\hline 341 & 0.994 & 0.627 & 0.877 & 0.825 & 0.667 & 0.726 & 0.277 & 0.714 & 0.994 \\
\hline 342 & 0.997 & 0.752 & 0.979 & 0.947 & 0.169 & 0.265 & 0.912 & 0.857 & 0.997 \\
\hline 343 & 1.000 & 0.877 & 0.662 & 0.662 & 0.574 & 0.571 & 0.186 & 1.000 & 1.000 \\
\hline
\end{tabular}

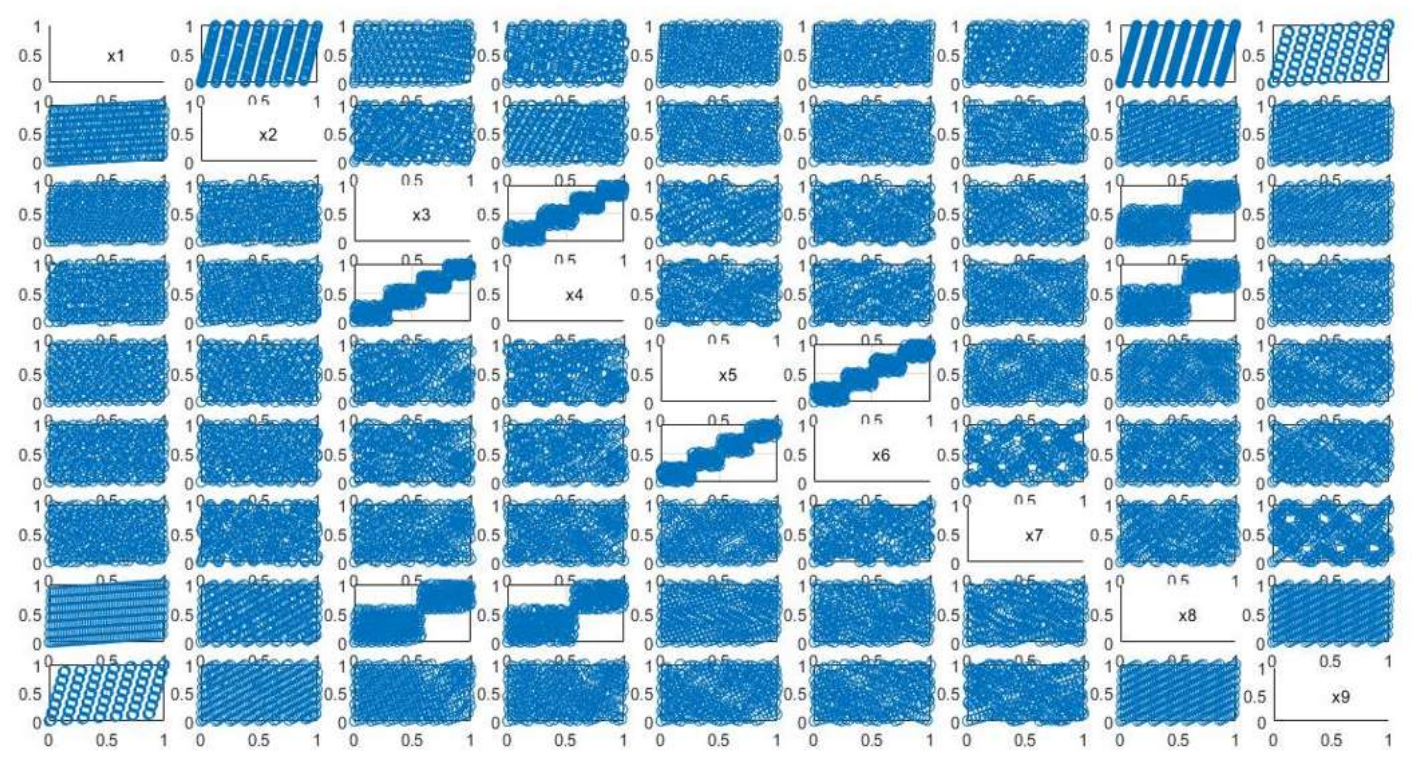

Figure 2: Projection properties of OA $(343,9)$ LHD 


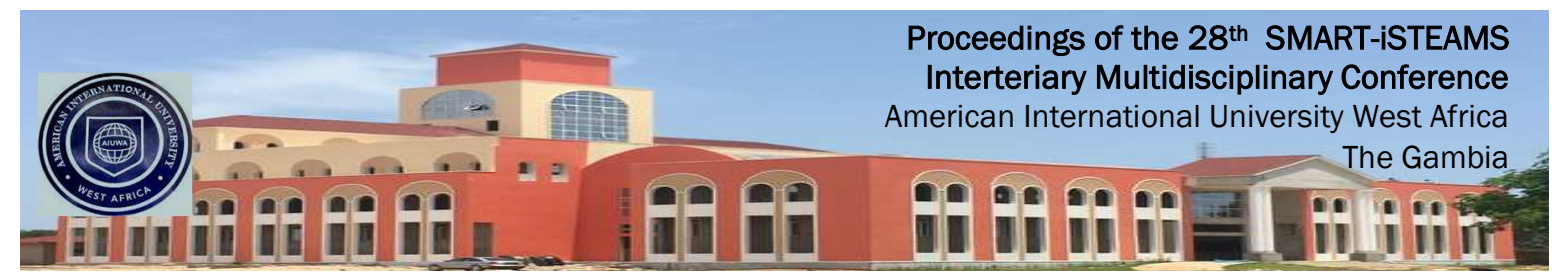

\section{APPLICATION}

The orthogonal array-based Latin hypercube design can be used to improve study design in biomedical research especially in the area of data obtained as the outcome of such research when parametric statistics is required to make inference about the study. For instance, using health related quality of life data of Children/Adolescent Living with HIV/AIDS in Lagos State discussed in the study conducted by Osuolale et al. (2020). The design points obtained for OA $(216,8)$ LHD were scaled accordingly with the minimum and maximum value in the respective health related quality of life (HRQL) data categorised into good (81.70 - 100), intermediate $(61.50-80.40)$ and poor (31.0 - 59.40) quality of life. The first three columns of OA $(216,8)$ LHD were used in this regard. Any thoughtful parametric statistical method can now be applied on the data presented in Table 3.

Table 3. Health Related Quality of Life Data

\begin{tabular}{|c|c|c|c|c|}
\hline No & GOOD & GOOD & INTERMEDIATE & POOR \\
\hline 1 & 81.77 & 84.99 & 61.58 & 31.11 \\
\hline 2 & 81.86 & 85.09 & 64.03 & 39.01 \\
\hline 3 & 81.94 & 85.16 & 66.47 & 46.90 \\
\hline 4 & 82.03 & 85.25 & 68.93 & 53.21 \\
\hline 5 & 82.12 & 85.34 & 71.38 & 37.05 \\
\hline 6 & 82.19 & 85.41 & 73.82 & 44.92 \\
\hline 7 & 82.29 & 85.51 & 64.11 & 35.06 \\
\hline 8 & 82.38 & 85.60 & 61.67 & 42.96 \\
\hline 9 & 82.45 & 85.67 & 69.02 & 50.06 \\
\hline 10 & 82.54 & 85.76 & 66.57 & 56.36 \\
\hline 11 & 82.62 & 85.84 & 73.92 & 33.10 \\
\hline 12 & 82.71 & 85.93 & 71.46 & 40.97 \\
\hline 13 & 82.80 & 86.02 & 66.66 & 39.15 \\
\hline 14 & 82.87 & 86.09 & 69.10 & 31.26 \\
\hline 15 & 82.96 & 86.18 & 61.75 & 53.35 \\
\hline 16 & 83.05 & 86.28 & 64.20 & 47.02 \\
\hline 17 & 83.13 & 86.35 & 76.28 & \\
\hline 18 & 83.22 & 86.44 & 78.38 & \\
\hline 19 & 83.31 & 86.51 & 69.19 & \\
\hline 20 & 83.38 & 86.60 & 66.74 & \\
\hline 21 & 83.48 & 86.70 & 64.30 & \\
\hline 22 & 83.55 & 86.77 & & \\
\hline 23 & 83.64 & 86.86 & & \\
\hline 24 & 83.73 & 86.95 & & \\
\hline 25 & 83.80 & 87.03 & & \\
\hline
\end{tabular}




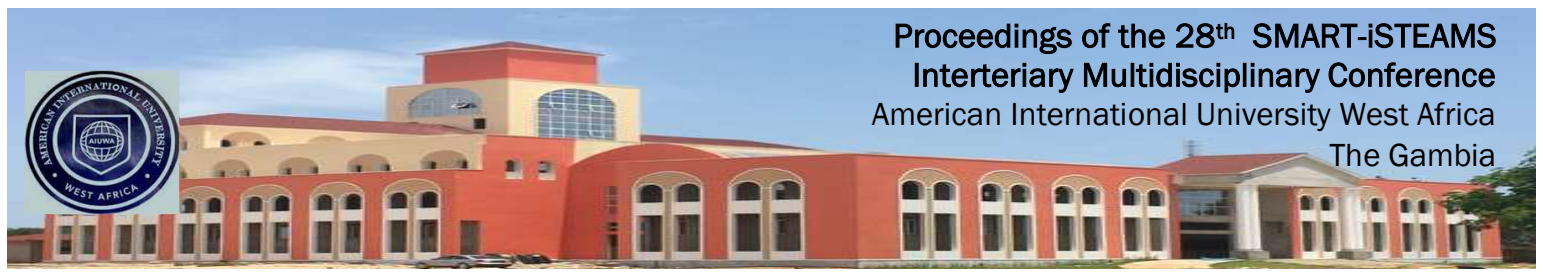

\begin{tabular}{|c|c|c|c|c|}
\hline No & GOOD & GOOD & INTERMEDIATE & POOR \\
\hline 26 & 83.90 & 87.12 & & \\
\hline 27 & 83.99 & 87.19 & & \\
\hline 28 & 84.06 & 87.28 & & \\
\hline 29 & 84.15 & 87.37 & & \\
\hline 30 & 84.23 & 87.45 & & \\
\hline 31 & 84.32 & 87.54 & & \\
\hline 32 & 84.41 & 87.63 & & \\
\hline 33 & 84.48 & 87.70 & & \\
\hline 34 & 84.57 & 87.79 & & \\
\hline 35 & 84.66 & 87.89 & & \\
\hline 36 & 84.74 & 87.96 & & \\
\hline 37 & 84.83 & 88.05 & & \\
\hline 38 & 84.90 & 88.12 & & \\
\hline
\end{tabular}

\section{DISCUSSION OF RESULTS}

The OA $(216,8)$ LHD contains 216 experimental runs (rows) and 8 factors (columns) while the OA $(343,9)$ LHD has 343 experimental runs (rows) and 9 factors (columns). The two cases of the OALHDs constructed have space-filling properties as depicted in Figures 1 and 2 and they achieve univariate stratification. Each column of the design can be used independently in application. The results presented in Table 3 gives the actual data for the health related quality of life for further analysis.

\section{CONCLUSION}

This study has shown how powerful orthogonal array-based Latin hypercube designs are in planning experiments and especially in improving the study design in biomedical research. The OA $(216,8)$ LHD and OA $(343,9)$ LHD constructed have better space-filling properties and they achieve uniformity in each dimension. This study concludes that the OALHDs constructed can be used whenever interest is focused on conducting either a scientific experiment or biomedical research. The data obtained in Table 3 gives a better picture of the health related quality of life to allow for statistical inference using a parametric statistical approach. The designs can also be applied for developing computer experiments.

\section{ACKNOWLEDGEMENTS}

The authors appreciate the support of the management of Nigerian Institute of Medical Research through conference attendance grant (GRANT NO: NM CAG-21-0001) awarded to the presenter to attend a conference in the Gambia. 


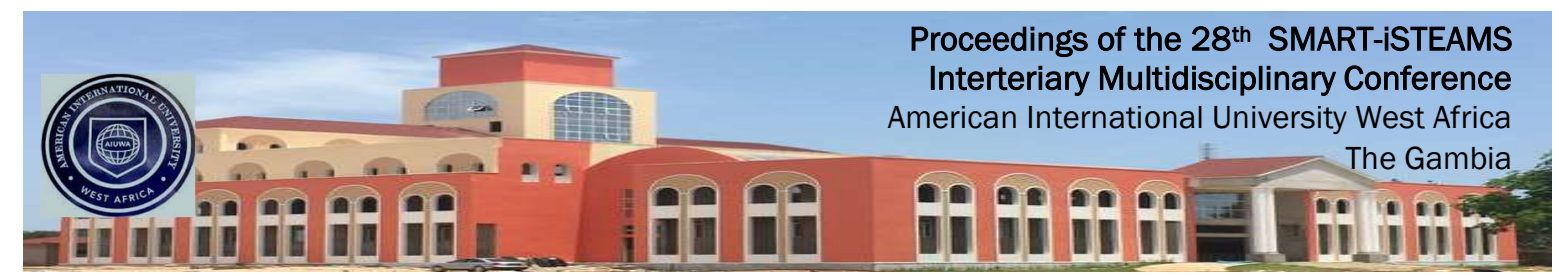

\section{REFERENCES}

Bose, R.C. \& Bush, K.A. (1952). Orthogonal arrays of strength two and three. Annals of Mathematical Statistics, 23, 508-524.

Currin, C., Mitchell, T., Morris, M. \& Ylvisaker, D. (1991). Bayesian prediction of deterministic functions with applications to the design and analysis of computer experiments.Journal of American Statistical Association, 86, 953-963.

Hedayat, A.S., Sloane, N.J.A. \& Stufken, J. (1999). Orthogonal arrays: Theory and applications. Springer-Verlag, New York.

Hernandez, A., Lucas, T. W. \& Carlyle, M. (2012). Constructing nearly orthogonal Latin hypercubes for any nonsaturated run-variable combination. ACM Trans. Model. Computer Simul.22 (4), Article 20, 1-17.

Iman, R. L. \& Conover, W. J. (1982). A distribution-free approach to inducingrank correlation among input variables.Communication in Statistics, Part B, 11,311-334.

Jin, R., Chen, W. \& Sudjianto, A. (2005). An efficient algorithm for constructing optimal design of computer experiments. Journal of Statistical Planning and Inference, 134, 268-287.

Johnson, M., Moore, L. \& Ylvisaker, D. (1990). Minimax and maximin distance design. Journal of Statistical Planning and Inference, 26, 131-148.

Joseph, V. R., Hung, Y. \& Sudjianto, A. (2008). Blind kriging: A new method for developing metamodels, ASME Journal of Mechanical Design, (130), 031102-1-8.

Leary, S., Bhaskar, A. \& Keane, A. (2003). Optimal orthogonal array-based Latin hypercubes. Journal of Applied Statistics, 30, 585-598. MR1969503.

Morris, M. D. \& Mitchell, T. J. (1995). Exploratory designs for computer experiments. Journal of Statistical Planning and Inference, 43, 381-402.

Osuolale, K. A., Yahya, W. B. \& Adeleke, B.L. (2014). Construction of space-filling designs for three input variables computer experiments. WASET, International Journal of Computer, Control, Quantum and Information Engineering, 8(9), 1624-1628.

Osuolale, K.A. (2017). Techniques for designing, modelling and analysing computer experiments (Ph.D Thesis). University of Ilorin, Nigeria.

Osuolale, K.A., Salako, A.O., Musa, A.Z. \& David, A.N. (2020). Health-related Quality of Life among Children/Adolescent Living with HIV/AIDS in Lagos State Using Analysis of Variance (ANOVA) Approach. Elixir Statistics 149, 55015-55019.

Owen, A.B. (1992). Orthogonal arrays for computer experiments, integration and visualization. Statistica Sinica, 2, 439-452.

Owen, A. B. (1994). Controlling correlations in Latin hypercube samples. Journal of the American Statistical Association, 89, 1571-1522.

Park, J.S. (1994). Optimal Latin hypercube designs for computer experiments. Journal of Statistical Planning and Inference, 39, 95-111.

Qian, Z., Seepersad, C., Joseph, R., Allen, J. \& Wu, C.F.J. (2006). Building surrogate models based on detailed and approximate simulations. ASME Transactions, Journal of Mechanical Design 128, 668-677.

Qian, P. Z. G., Tang, B. \& Wu, C. F. J. (2009). Nested space-filling designs for computer experiments with two levels of accuracy. Statistica Sinica, 19, 287-300.

Rao, C.R. (1947). Factorial experiments derivable from combinatorial arrangements of arrays. Journal of the Royal Statistical Society (Suppl.), 9, 128-139. 


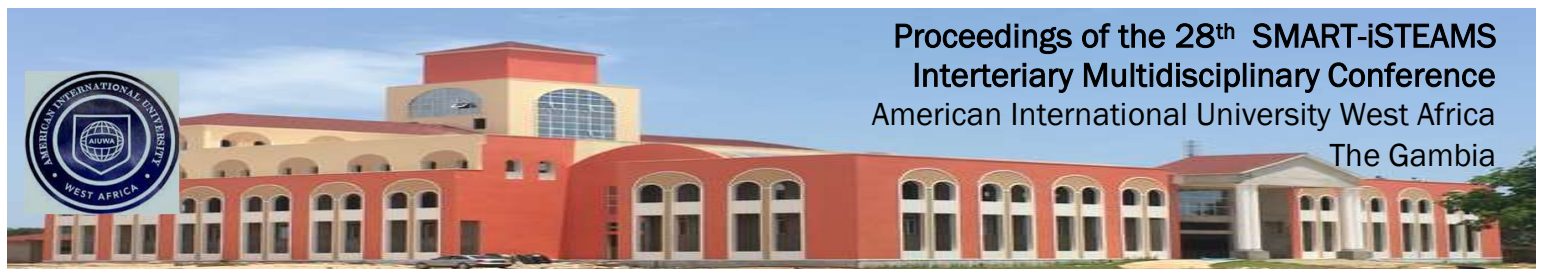

Shewry, M.C. \& Wynn, H.P. (1988). Maximum entropy sampling with application to simulation codes. Proceedings of the 12th World Congress on Scientific Computation, IMAC88.

Tang, B. (1993). Orthogonal array-based Latin hypercubes. Journal of the American Statistical Association, 88, 1392-1397.

Wu, C. F. J. \& Hamada, M. (2000). Experiments: Planning, analysis and parameter design optimization. Wiley, New York.

Yahya, W.B. \& Osuolale, K.A. (2016). A method of constructing orthogonal array-based Latin hypercube designs for computer experiments. International Journal of Hybrid Information Technology, 9(6), 253-262.

Ye, K. Q. (1998). Orthogonal column Latinhypercubes and their application in computer experiments.Journal of the American Statistical Association, 93(444), 1430-1439. Retrieved from http://dx.doi.org/10.1080/01621459.1998.10473803.

Ye, K.Q., Li, W. \& Sudjianto, A. (2000). Algorithmic construction of optimal symmetric Latin hypercube deisgns. Journal of Statistical Planning and Inference, 90, 145-159. 